\title{
Tendência Genética para a Produção de Leite e de Gordura em Rebanhos da Raça Holandesa no Estado de Minas Gerais
}

\author{
Marcus Cordeiro Durães ${ }^{1}$, Ary Ferreira de Freitas ${ }^{1}$, José Valente ${ }^{1}$, Nilson Milagres Teixeira ${ }^{1}$, \\ Ricardo Bertola Barra ${ }^{2}$
}

\begin{abstract}
RESUMO - O objetivo deste trabalho foi estimar as tendências genéticas para a produção de leite e gordura nos rebanhos da raça Holandesa do Estado de Minas Gerais. As análises estatísticas foram realizadas por intermédio da metodologia dos modelos mistos, usando-se máxima verossimilhança restrita e modelo animal. Os efeitos fixos considerados no modelo foram: rebanho-ano-estação de parto e os efeitos linear e quadrático da idade da vaca ao parto. Os efeitos aleatórios foram animal e erro. A tendência genética foi calculada pela regressão do valor genético sobre o ano de nascimento da vaca; nesta análise, foram usados 18.482 registros de produção de leite e gordura de vacas de primeira cria, nascidas de 1986 a 1996. As estimativas de ganho genético para produção de leite e gordura foram 18,4 e $0,6 \mathrm{~kg}$, respectivamente. A média de produção de leite e gordura aumentou anualmente, variando de $5083 \pm 50$ a $6876 \pm 51 \mathrm{~kg}$, e de 172,6 \pm 1,7 a 228,5 $\pm 1,7 \mathrm{~kg}$, respectivamente. Os valores genéticos estimados foram negativos em 1986 e 1987 e positivos após este período, com aumento acentuado nos últimos anos, indicando que os produtores de leite de Minas Gerais vêm adotando critérios para aumentar a capacidade de produção de seus rebanhos.
\end{abstract}

Palavras-chave: máxima verossimilhança, modelo animal, parâmetros genéticos

\section{Genetic Trend for Milk and Fat Productions for Holstein Cattle in Minas Gerais State}

\begin{abstract}
The objective of this work was to estimate the genetic trends for milk and fat yields of Holstein Dairy Cattle, in Minas Gerais State, Brazil. The statistical analyses were made by means of mixed models, using restricted maximum likelihood and animal model. The fixed effects studied were: herd-year-season at calving and linear and quadratic effect of age at calving besides the animal and residual random effects. The genetic trends were obtained by regression of the breeding value on the year of birth of cows. Records used in the analyses were 18,482 first lactations of cows born from 1986 to 1996 . The genetic trends for milk and fat yield were 18.4 and $0.6 \mathrm{~kg}$, respectively. The average of milk and fat yields were $5083 \pm 50$ to $6876 \pm 51 \mathrm{~kg}$, and from $172.6 \pm 1.7$ to $228.5 \pm 1.7 \mathrm{~kg}$, respectively. The breeding values estimated were negative in 1986 and 1987, and positive thereafter, with accentuate increase in the last years. This is an indication that milk farmers of Minas Gerais State have been taking strategies in order to increase the milk productions.
\end{abstract}

Key Words: animal model, genetic parameters, maximum likelihood

\section{Introdução}

Desde a década de 50, em várias partes do mundo, vêm sendo divulgadas estimativas de ganhos genéticos anuais ou tendências genéticas, empregando-se diferentes metodologias de estimação. Os valores encontrados, em geral, variam com a metodologia empregada.

Alguns estudos procuravam avaliar as diferenças no desempenho produtivo de vacas filhas de touros utilizados em inseminação artificial, em relação aos de monta natural. Tudo indica que a inseminação artificial, por meio da utilização de sêmen de touros provados, produziu filhas mais produtivas. Esta técnica de reprodução animal tem viabilizado o aumento da capacidade produtiva de diversos rebanhos, mesmo em condições tropicais.

LEGATES (1992) analisou os dados de um programa de melhoramento de bovinos leiteiros iniciado em 1949, envolvendo inseminação artificial e monta natural, com duração de 25 anos. O autor concluiu que a produção de leite aumentou de 4536 para $7711 \mathrm{~kg}$ e a porcentagem de gordura, de 3,45 para $3,75 \mathrm{~kg}$ no período estudado. $O$ ganho genético estimado variou de 29 a $46 \mathrm{~kg}$ por ano, representando cerca de $25 \%$ do total do aumento de produção no período. Segundo WATTIAUX (1995), o ganho genético dos rebanhos holandeses dos Estados Unidos foi de apenas $1000 \mathrm{~kg}$ entre 1965 e 1985 e o ganho genético esperado de 1985 até o ano 2005 poderá ser aproximadamente de

\footnotetext{
1 Pesquisador da Embrapa Gado de Leite. Rua Eugênio do Nascimento, 610, Dom Bosco, Juiz de Fora, MG, CEP: $36038-330$. Bolsista do CNPq. E-mail: dcm075@cnpgl.embrapa.br

2 Superintendente Técnico da Associação de Criadores de Gado Holandês de Minas Gerais
} 
$6000 \mathrm{~kg}$, graças às modernas tecnologias disponíveis, que aumentaram a precisão na identificação dos animais geneticamente superiores.

POWELL e WIGGANS (1991) avaliaram o ganho genético para a produção de leite de rebanhos da raça Holandesa do México, usando modelo animal em 123.397 lactações de 50.538 vacas e 4573 touros. O ganho genético estimado, ajustando uma curva quadrática para os valores genéticos médios sobre ano de nascimento, foi de $87 \mathrm{~kg}$. Porém, TASSELL et al. (1991), trabalhando com dados de outros rebanhos, estimaram em $34,9 \mathrm{~kg}$ de leite/ano o ganho genético baseado no diferencial de seleção e intervalo de gerações de todos os anos e, nos últimos cinco anos, as vacas classificadas como "grade" tiveram ganho genético médio de $57,2 \mathrm{~kg}$. Os autores concluíram que o ganho observado foi menor que o esperado (96 kg por ano), em virtude dos longos intervalos de geração, e os diferenciais de seleção, menores que os teoricamente possíveis.

BURNSIDE et al. (1992) analisaram os dados coletados de 1972 a 1988 de rebanhos da raça Holandesa da Itália, utilizando modelo animal para estimar os valores genéticos para leite, gordura e proteína. Verificaram que o ganho genético anual para essas características, estimado pela mudança da média do valor genético sobre o ano de nascimento, aumentou de 0,08 desvios-padrão até 1984 e de 0,25 a 0,30 de 1985 a 1988.

Segundo SASAKI et al. (1993), utilizando a metodologia dos modelos mistos e a máxima verossimilhança restrita (REML), o aumento anual da produção de leite foi de $303,7 \mathrm{~kg}$ para as vacas multíparas e de $126,5 \mathrm{~kg}$ para as primíparas da raça Holandesa. A tendência estimada para o ganho genético anual para a produção de leite foi de $15,5 \mathrm{~kg}$, no período de 1974 a 1989.

TEIXEIRA et al. (1994) estimaram em 94 a $139 \mathrm{~kg}$ o ganho genético dos rebanhos da raça Holandesa no Estado do Paraná, empregando dois métodos para estimar o ganho genético.

Na Córeia do Sul, KIM et al. (1999) estimaram as tendências fenotípicas e genotípicas em 126,5 e $35,0 \mathrm{~kg}$ em rebanhos da raça Holandesa. As médias da produção de leite e gordura foram de $7.466 \pm 1.881 \mathrm{~kg}$ e $267 \pm 70 \mathrm{~kg}$, respectivamente.

As estimativas do progresso genético são de grande importância nos programas de melhoramento genético animal. As Associações de Criadores, como entidades representativas dos produtores, necessitam monitorar e avaliar o progresso genético dos rebanhos de seus associados, no sentido de orientá-los na melhor utilização dos recursos genéticos existentes. Portanto, o objetivo do presente estudo foi estimar a tendência genética da produção de leite e gordura para os rebanhos da raça Holandesa do Estado de Minas Gerais.

\section{Material e Métodos}

Foram utilizados 18.482 registros de produção de vacas de 359 rebanhos obtidos do controle leiteiro realizado pelos técnicos da Associação de Criadores de Gado Holandês de Minas Gerais, das vacas Holandesas de primeira lactação, nascidas de 1986 a 1996.

As análises estatísticas foram realizadas por intermédio da metodologia dos modelos mistos, usando-se máxima verossimilhança restrita em um modelo animal. O modelo empregado para análise estatística foi o seguinte:

$$
\mathrm{Y}_{\mathrm{ij} 1}=\mathrm{m}+\mathrm{A}_{\mathrm{i}}+\mathrm{RAE}_{\mathrm{j}}+\mathrm{ID}_{1}+\mathrm{ID}_{1}^{2}+\mathrm{e}_{\mathrm{ij} 1}
$$

em que $\mathrm{Y}_{\mathrm{ijl}}$ é a produção de leite ou de gordura ao primeiro parto, da vaca i, medido dentro do rebanhoano-estação j, com os efeitos linear e quadrático da idade da vaca em dias, sendo $\mathrm{m}$ uma constante comum a toda população e $\mathrm{e}_{\mathrm{ijl}} \mathrm{o}$ efeito residual comum aos ${ }_{i j l}$ ésimo registros de produção. $A_{i}$ é o efeito genético do animal, no caso, a vaca i considerado aleatório; $\mathrm{RAE}_{\mathrm{j}}$, o efeito fixo de rebanho-anoestação j ao parto, j variando de 1 a 1921, sendo consideradas duas estações: seca (abril - setembro) e águas (outubro - março). $\mathrm{ID}_{1}$ e $\mathrm{ID}_{1}^{2}$ representam os efeitos linear e quadrático da idade da vaca ao parto em dias. Aplicando-se o mesmo modelo, analisaramse os dados coletados de 1986 a 1991 (8346 observações) e de 1992 a 1996 (10.136 observações).

As estimativas das tendências genéticas de leite e gordura foram obtidas pela regressão do valor genético médio ponderada pelo número de observações sobre o ano de nascimento da vaca.

\section{Resultados e Discussão}

Os dados foram divididos em dois grupos. O primeiro, formado por dados de controle leiteiro coletados de 1986 a 1991 e o segundo, por dados coletados de 1992 a 1996. No primeiro caso, as estimativas da tendência genética anual para leite e gordura e a média da produção de leite, envolvendo 8346 observações, foram de apenas $8,4,0,28$ e $5373 \mathrm{~kg}$, respectivamente, enquanto no segundo, envolvendo 10.136 observações, foi de 16,8, 0,68 e $6346 \mathrm{~kg}$.

A média de produção de leite, $6346 \mathrm{~kg}$ calculada 
para o segundo período, foi cerca de $18 \%$ acima da média $5373 \mathrm{~kg}$, observada no primeiro. As estimativas de tendência genética para leite e gordura foram maiores no segundo período, refletindo maior pressão de seleção na escolha de reprodutores, em que os produtores de leite deram mais ênfase ao aumento da capacidade de produção de leite das vacas de seus rebanhos. Este fato demonstra a preocupação dos produtores de leite, na última década, com a melhoria não somente das condições de meio, mas também da qualidade genética de seus animais.

Na Tabela 1 são apresentados o número de observações, a média dos valores genéticos preditos e de produção anual, com os respectivos errospadrão, e os valores mínimo e máximo das produções de leite, de acordo com o ano de nascimento da vaca. Na Tabela 2, as mesmas informações para a produção de gordura, levando-se em consideração o conjunto de dados coletados de 1986 a 1996, podem ser visualizadas.

O ganho genético anual para a produção de leite e gordura foi de 18,4 e $0,6 \mathrm{~kg}$, respectivamente. Estes valores são relativamente baixos, quando comparados aos resultados observados na literatura. Por exemplo, TEIXEIRA et al. (1994) apresentaram ganhos genéticos anuais variando de 94 a $128 \mathrm{~kg}$ para os rebanhos da raça Holandesa do Estado do Paraná. Contudo, os autores alegaram que esses valores eram bastante elevados comparados àqueles encontrados na literatura. Ponderaram, ainda, que os dados deveriam ser novamente analisados utilizando-se a mesma metodologia empregada no presente trabalho, que não foi utilizada naquela época, pelas dificuldades computacionais existentes.
As médias das produções de leite variaram, no período de 1986 a 1996 , de $5083 \pm 50$ a $6876 \pm 51 \mathrm{~kg}$. Observou-se que houve aumento da média de produção de leite ano após ano, durante o período estudado. $\mathrm{O}$ aumento da média de produção de leite observado foi de $1793 \mathrm{~kg}$ em 11 anos, ou seja, aumento médio de $163 \mathrm{~kg}$ por ano. O acréscimo da produção anual, descrito por SASAKI et al. (1993) foi de $126,5 \mathrm{~kg}$, e o ganho genético foi estimado em $15,5 \mathrm{~kg}$, o que revela serem estes valores inferiores aos observados no presente trabalho. Todavia, TEIXEIRA et al. (1994), POWELL e WIGGANS (1991) e TASSELL et al. (1991) encontraram valores de tendência genética superiores aos do presente estudo. Contudo, KIM et al. (1999) relataram ganho genético de apenas $0,47 \%$ e comentaram que esse valor é menor que os $2 \%$ teoricamente possível de serem alcançados. Essas discrepâncias encontradas podem ser causadas por diferentes fatores, como, por exemplo, falha na escolha de reprodutores, utilização de sêmen de touros de baixa confiabilidade (abaixo de 70\%), de menor preço, com o objetivo de reduzir os custos investidos na compra de sêmen ou mudança no enfoque de seleção. Isto pode ocorrer quando os produtores enfatizam diversas características no programa de seleção. De fato, é comum os produtores selecionarem para o aumento da produção de leite e outras características de produção, como a gordura, a proteína, além de características de tipo. Daí, a necessidade e a importância de se realizarem estudos periódicos para monitorar o ganho genético de rebanhos leiteiros, principal-

Tabela 1 - Número de observações, média dos valores genéticos preditos e da produção de leite e os respectivos erros-padrão, os valores mínimo e máximo da produção de leite, por ano de nascimento da vaca

Table 1 - Number of observations, average breeding values and average milk yield and respective standard errors (se), minimum and maximum values for milk yields, according to year of birth of the cow

\begin{tabular}{lccccc}
\hline $\begin{array}{c}\text { Ano } \\
\text { Year }\end{array}$ & $\begin{array}{c}\text { Número } \\
\text { Number }\end{array}$ & $\begin{array}{c}\text { MVGL }^{\mathrm{a}} \pm \mathrm{EP}^{\mathrm{c}} \\
\text { Average } B V \pm S E\end{array}$ & $\begin{array}{c}\text { MPRODL }^{\mathrm{b}} \text { EP } \\
\text { Av. milk yield } \pm \text { SE }\end{array}$ & $\begin{array}{c}\text { Mínimo } \\
\text { Minimum }\end{array}$ & $\begin{array}{c}\text { Máximo } \\
\text { Maximum }\end{array}$ \\
\hline 1986 & 803 & $-9,0 \pm 3,4$ & $5083 \pm 50$ & 2064 & 10.907 \\
1987 & 1286 & $-0,9 \pm 3,3$ & $5173 \pm 40$ & 1904 & 10.482 \\
1988 & 1491 & $2,0 \pm 3,3$ & $5100 \pm 39$ & 1814 & 12.853 \\
1989 & 1350 & $3,8 \pm 3,5$ & $5317 \pm 43$ & 1822 & 11.920 \\
1990 & 1608 & $16,0 \pm 3,5$ & $5593 \pm 40$ & 1981 & 12.946 \\
1991 & 1808 & $36,0 \pm 3,5$ & $5713 \pm 38$ & 1844 & 13.211 \\
1992 & 2273 & $83,0 \pm 3,3$ & $6142 \pm 37$ & 1740 & 14.108 \\
1993 & 2317 & $90,0 \pm 3,4$ & $6373 \pm 3433$ & 1882 & 12.208 \\
1994 & 2356 & $130 \pm 3,2$ & $6448 \pm 35$ & 1556 & 15.390 \\
1995 & 2121 & $135,0 \pm 3,4$ & $6876 \pm 51$ & 1634 & 15.801 \\
1996 & 1069 & $139,0 \pm 4,5$ & 13.622 \\
\hline
\end{tabular}

a MVGL = Média dos valores genéticos preditos para leite. ${ }^{\mathrm{b} M P R O D L}=$ Média da produção de leite. ${ }^{\mathrm{C}} \mathrm{EP}=$ erro-padrão; (SE= standard error). 
DURÃES et al.

Tabela 2 - Número de observações, média dos valores genéticos preditos e os respectivos erros-padrão, os valores mínimos e máximos de gordura, por ano de nascimento

Table 2 - Number of observations, fat genetics predicted values and production average with respectives standard erros, minimum and maximum values for fat yields, accordingly to year of birth

\begin{tabular}{|c|c|c|c|c|c|}
\hline $\begin{array}{l}\text { Ano } \\
\text { Year }\end{array}$ & $\begin{array}{l}\text { Número } \\
\text { Number }\end{array}$ & $\begin{array}{c}\mathrm{MVGG}^{\mathrm{a}} \pm \mathrm{EP}^{\mathrm{c}} \\
\text { Average } B V \pm S E\end{array}$ & $\begin{array}{l}\mathrm{MPRODG}^{\mathrm{b}} \pm \mathrm{EP} \\
\text { Av. milk yield } \pm S E\end{array}$ & $\begin{array}{l}\text { Mínimo } \\
\text { Minimum }\end{array}$ & $\begin{array}{r}\text { Máximo } \\
\text { Maximum } \\
\end{array}$ \\
\hline 1986 & 803 & $-0,4 \pm 0,1$ & $172,6 \pm 1,7$ & 71 & 401 \\
\hline 1987 & 1286 & $-0,1 \pm 0,1$ & $176,6 \pm 1,4$ & 64 & 357 \\
\hline 1988 & 1491 & $0,7 \pm 0,1$ & $174,3 \pm 1,3$ & 61 & 401 \\
\hline 1989 & 1350 & $0,2 \pm 0,1$ & $179,6 \pm 1,4$ & 55 & 441 \\
\hline 1990 & 1608 & $0,3 \pm 0,1$ & $186,6 \pm 1,3$ & 61 & 521 \\
\hline 1991 & 1808 & $1,1 \pm 0,1$ & $190,3 \pm 1,2$ & 71 & 443 \\
\hline 1992 & 2273 & $2,2 \pm 0,1$ & $204,5 \pm 1,2$ & 22 & 503 \\
\hline 1993 & 2317 & $2,7 \pm 0,1$ & $206,3 \pm 1,1$ & 47 & 434 \\
\hline 1994 & 2356 & $4,1 \pm 0,1$ & $214,3 \pm 1,1$ & 27 & 482 \\
\hline 1995 & 2121 & $4,4 \pm 0,1$ & $216,6 \pm 1,2$ & 49 & 592 \\
\hline 1996 & 1069 & $4,4 \pm 0,1$ & $228,5 \pm 1,7$ & 85. & 446 \\
\hline
\end{tabular}

mente nos países localizados nas regiões tropicais, tradicionais importadores de sêmen de touros testados nas regiões temperadas.

A produção de gordura também aumentou continuamente, variando de 172,6 kg em 1986 a $228,5 \mathrm{~kg}$ em 1996. Este aumento de produção reflete o aumento ocorrido na produção de leite, ou seja, acréscimo médio anual em torno de $5 \mathrm{~kg}$.

As produções de leite e de gordura são os reflexos da escolha dos touros utilizados em inseminação artificial. Pela Tabela 1, pode-se verificar que a média do valor genético estimado para leite e gordura foi maior com o decorrer dos anos, ou seja, foi negativa em 1986 e 1987, tanto para leite quanto para gordura, e positiva a partir de 1988 até 1996, com maiores valores nos últimos anos, refletindo alguma melhoria genética obtida nesse período.

A produção de leite variou de um mínimo de 1509 em 1991 a um máximo de $15.801 \mathrm{~kg}$ em 1995. As produções mínima e máxima de gordura foram $22 \mathrm{e}$ $592 \mathrm{~kg}$, respectivamente. Essa variação indica que os controles leiteiros de gado holandês do Estado de Minas Gerais estão sendo feitos nas vacas de alta e baixa produção. O baixo valor da tendência genética observado para leite como para gordura reflete a escolha do sêmen utilizado pelos produtores, de 1986 a 1996, assim como os critérios de alimentação e manejo dos rebanhos sob controle leiteiro.

\section{Conclusões}

A média dos valores preditos para leite e gordura foram menores na década de 80 e substancialmente maiores na década de 90 , demonstrando que os criadores do Estado de Minas Gerais vêm envidando esforços para aumentar a capacidade de produção de seus rebanhos.

Contudo, em virtude da baixa tendência genética tanto para leite quanto para gordura observada, pode-se deduzir que os aumentos das médias de produção ocorreram principalmente pela melhoria de manejo e alimentação dos animais.

Todavia, pode-se concluir que na década de 90 os produtores selecionaram touros com maior capacidade de transmissão para características produtivas, em relação aos utilizados na década de 80 , refletindo o interesse no aumento da capacidade de produção de seus animais.

Porém, apesar da disponibilidade de sêmens importados de touros provados no mercado nacional, a escolha e o uso deles são feitos com pouca ênfase em características de produção, possivelmente pela inclusão de características não produtivas, como as de conformação, no programa de seleção.

Portanto, o monitoramento periódico de rebanhos leiteiros torna-se importante para avaliar a tendência genética ocorrida no período e serve para alertar os produtores sobre a necessidade de reavaliar o seu programa de seleção. 


\section{Referências Bibliográficas}

BURNSIDE, E.B., JANSEN, G.B., CIVATI, G. et al. 1992. Observed and theoretical genetics trends in a large dairy population under intensive selection. J. Dairy Sci., 75(8):2242-2253.

LEGATES, J.E. 1992.The North Carolina institucional breeding program; an experiment in dairy cattle improvement. North Carolina Agricultural Research. Technical Bulletin 297. 15p.

KIM, J.S., PARK, K.D., JEONG, H.Y. et al. 1999. Estimation of regional genetic trends for milk and fat yields in the Korean Holstein population. Korean J. Anim. Sci., 41(1): 11-14.

POWELL, R.L.,WIGGANS, G.R. 1991. Animal model evaluations for Mexican Holsteins. J. Dairy Sci., 74(4):1420-1427.

SASAKI, O., TOGASHI, K., YOKOUCHI, K. et al. 1993. Analysis of annual genetic gain in milk yield in a dairy herd. Research bulletin of the Hokkaido Agricultural Experiment Station. 158. p.21-30.
TASSELL, C.P., VAN VLECK, L.D. 1991. Estimates of genetic selection differentials and generations interval for four paths of selection. J. Dairy Sci., 74(3):1.078-1.086.

TEIXEIRA, N. M., FREITAS, A.F., RIBAS, N.P., et al. 1994. Tendências genéticas em rebanhos da raça Holandesa no Estado do Paraná. I. Produção de leite. R. Bras. Zootec., 23(6):983-991.

WATTIAUX, M.A. 1995. Reproduction and genetic selection. In: TECHNICAL dairy guide. Madison: University of Wisconsin. p.111-145.

Recebido em: 07/06/00

Aceito em: 15/09/00 